\title{
Insulin clearance: an underappreciated modulator of plasma insulin concentration
}

\author{
Sun H Kim, Gerald M Reaven
}

Department of Medicine,

Stanford University School of Medicine, Stanford,

California, USA

\section{Correspondence to}

Dr Sun H Kim, Department of Medicine, Stanford University Medical Center, 300 Pasteur Drive, Room S025, Stanford, CA 94305-5103, USA; sunhkim@stanford.edu

Accepted 4 May 2016 Published Online First 26 May 2016

Copyright () 2016 American Federation for Medical Research
CrossMark

To cite: Kim SH, Reaven GM. J Investig

Med 2016;64:1162-

1165.

\section{ABSTRACT}

Plasma glucose concentrations are tightly regulated and maintained within a narrow range in nondiabetic individuals. Maintenance of this physiological state is primarily a function of the ability of the pancreatic $\beta$-cells to modify insulin secretion rate (ISR), thus preventing wide-swings in plasma glucose concentrations. As a consequence, and in contrast to plasma glucose concentrations, plasma insulin concentrations vary substantially in non-diabetic individuals. Although differences in ISR are primarily responsible for the variability in plasma insulin concentration, there is increasing evidence that differences in insulin clearance rate (ICR) also play a role in regulation of plasma insulin concentration. The goal of this mini-review is to highlight situations that demonstrate the important role of ICR in both insulin and glucose homeostasis.

\section{INTRODUCTION}

A major physiological factor affecting plasma insulin concentrations among non-diabetic persons is differences in insulin-mediated glucose disposal. Insulin sensitivity quantified in this manner can vary up to sixfold in nondiabetic individuals, ${ }^{1}$ and insulin sensitivity (or resistance) affects plasma insulin concentration by modulating insulin secretion rate (ISR). Specifically, by using an experimental approach based on determining the response to graded intravenous glucose infusions, we have demonstrated that the dose-response relationship between plasma glucose concentration and ISR is shifted to the left in insulin-resistant as compared with insulin-sensitive individuals. ${ }^{2}$ As a consequence, insulin-resistant individuals secrete more insulin at any given plasma glucose concentration than their insulinsensitive counterparts, leading to higher insulin concentrations in insulin-resistant versus insulin-sensitive individuals without diabetes.

On the other hand, results of our graded glucose infusion studies also demonstrated that, when individuals at extremes of insulin sensitivity are compared, the plasma insulin concentrations approximately double; however, ISR is only $\sim 50 \%$ higher. $^{2}$ Thus, something other than an increase in ISR had to have played a role in the accentuated increase in plasma insulin concentration in the insulin-resistant persons. That 'something other' was a decrease in insulin clearance rate (ICR), which also varies with degree of insulin resistance, thereby contributing to the hyperinsulinemia characteristic of insulin-resistant, non-diabetic individuals.

\section{ICR IS INVERSELY ASSOCIATED WITH INSULIN RESISTANCE}

Figure 1 is based on unpublished data from our research group and illustrates the significant inverse relationship $(\mathrm{r}=-0.45, \mathrm{p}<0.001)$ between insulin resistance and ICR in 276 Caucasian individuals without diabetes (mean age $52.8 \pm 8.7$ years, mean body mass index (BMI) $30.5 \mathrm{~kg} / \mathrm{m}^{2} \pm 3.5$ ). Insulin resistance was measured by quantifying the steady-state plasma glucose (SSPG) concentration during the insulin suppression test. ${ }^{3}$ Higher SSPG concentration reflects greater degree of insulin resistance and lower insulin-mediated glucose uptake in the skeletal muscle. ICR was calculated by dividing the insulin infusion rate by the steady-state plasma insulin concentration during the insulin suppression test. As octreotide is used to suppress endogenous ISR during the insulin suppression test, this approach provides a precise estimate of exogenous ICR. Figure 1 emphasizes the great variability in insulin sensitivity, and documents the fact that the more insulin resistant an individual, the lower the ICR. Furthermore, the ability of changes in ICR to modulate plasma insulin concentrations is not trivial and, based on results in a recent study, it appears that changes in ICR can account for $\sim 25 \%$ of the variability in plasma insulin concentration. ${ }^{4}$

The data in figure 1 could be viewed as an example of a teleologically useful compensatory mechanism to minimize the adverse impact of resistance to insulin-mediated glucose disposal. The physiological response of the pancreatic $\beta$-cells to insulin resistance is to increase the ISR in order to attain the compensatory hyperinsulinemia necessary to maintain normal glucose tolerance. A concomitant decrease in ICR would complement the increase in ISR in achieving the plasma insulin concentration required to prevent loss of glucose tolerance in insulin-resistant individuals.

Another possibility, and a paradoxical one, is the notion that an adverse consequence of insulin resistance, specifically hepatic steatosis, would help maintain the degree of hyperinsulinemia needed to prevent hyperglycemia in insulin-resistant individuals. ${ }^{5} 6$ For example, Korenblat et $a l^{5}$ showed, in obese individuals, 


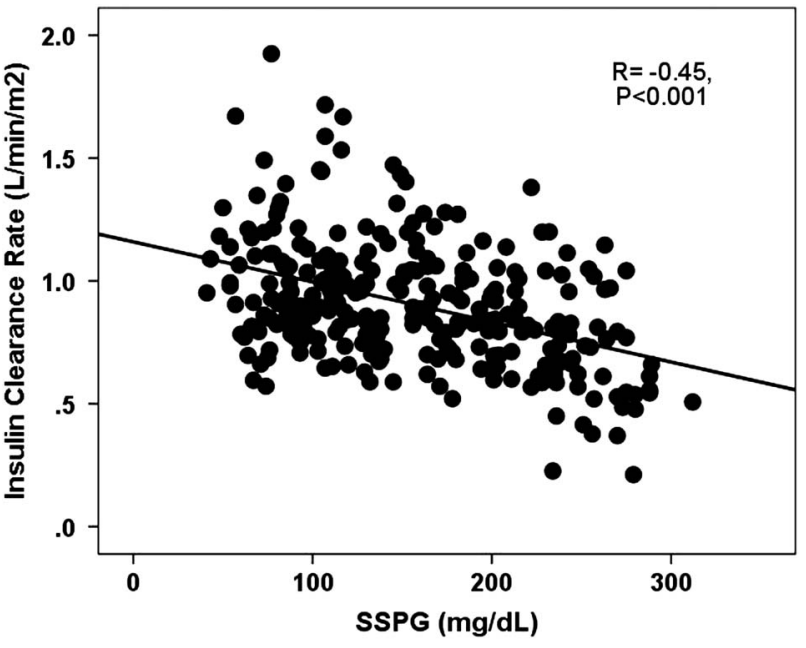

Figure 1 Relationship between insulin resistance (steady-state plasma glucose, SSPG) and insulin clearance rate, measured during the insulin suppression test.

that intrahepatic fat as measured by MR spectroscopy varied by 65 -fold from $0.7 \%$ to $45.5 \%$, whereas BMI only varied by 1.5 -fold $\left(30-46 \mathrm{~kg} / \mathrm{m}^{2}\right) .{ }^{5}$ Roughly half of the variance in intrahepatic fat was explained by differences in insulin sensitivity as measured by the hyperinsulinemic-euglycemic clamp $(r=-0.69, p<0.001)$. The liver is the major site for insulin clearance, ${ }^{7}$ and hepatic steatosis has been shown to negatively correlate with ICR. ${ }^{8}$ In vitro studies have shown ICR to be lower in hepatocytes loaded with triglycerides than in normal hepatocytes. ${ }^{9}$ Human studies have also shown decrease in ICR associated with increase in intracellular triglyceride content. ${ }^{8}$ Therefore, increases in intrahepatic fat may mediate the decrease in ICR associated with insulin resistance, and inadvertently help maintain glucose homeostasis by contributing to compensatory hyperinsulinemia.

\section{IMPROVEMENT IN INSULIN SENSITIVITY INCREASES ICR}

Although the inverse association between insulin resistance and ICR is clear from figure 1, the directional link between the two has not been well studied. Although insulin resistance likely leads to decrease in ICR and increase in plasma insulin concentration, others also have argued that hyperinsulinemia can lead to insulin resistance. ${ }^{10}$ Thus we have evaluated whether improving insulin resistance can change ICR, as well as ISR, by administering rosiglitazone, an insulin sensitizer, for 12 weeks to insulin-resistant individuals without diabetes. Rosiglitazone led to a $27 \%$ improvement in insulin sensitivity (SSPG concentration decreased from 243 to $177 \mathrm{mg} / \mathrm{dL}, \mathrm{p}<0.001) .{ }^{11} \mathrm{ISR}$, quantified by the graded glucose infusion method, and plasma insulin concentration, both significantly decreased in association with the enhanced insulin sensitivity in rosiglitazonetreated persons. In contrast, ICR, measured both during the insulin suppression test (exogenous insulin clearance) and during graded intravenous infusions of glucose (endogenous insulin clearance), increased, by $34 \%$ and $21 \%$, respectively. Therefore, improving insulin resistance directly affected ICR.

Obviously, the increase in ICR would contribute to the decrease in plasma insulin concentration needed to prevent hypoglycemia in the context of the drug-induced decrease in insulin resistance. On the other hand, similar to the association of the decrease in ICR with increased insulin resistance, the physiological mechanism accounting for the change in ICR remains to be defined. However, although we did not assess hepatic fat in our study, rosiglitazone has been shown to decrease intrahepatic triglyceride content. ${ }^{12}$ Therefore, whether the increase in ICR was secondary to a decrease in hepatic steatosis, or the consequence of a downstream effect of improving insulin resistance, remains to be seen.

\section{WEIGHT LOSS INCREASES ICR}

We have demonstrated, in a group of obese women, varying dramatically in degree of insulin resistance, that losing $\sim 10 \%$ of initial body weight was not associated with overall improvement in insulin sensitivity. ${ }^{13}$ The failure to discern a weight-loss-associated improvement in insulin sensitivity is most likely due to the fact that the study group included substantial numbers of insulin-sensitive, obese participants, in whom weight loss has little, if any, effect on insulin action. ${ }^{14}$ However, in that same study, we were able to demonstrate that plasma insulin concentration fell, and this was associated with an increase in ICR. ${ }^{13}$ Interestingly, no significant changes were detected in ISR. Other studies also have shown greater changes in ICR than ISR during modest $(<10 \%)$ weight loss ${ }^{15}$ and weight gain. $^{16}$

Therefore, ICR may adapt more readily to changes in insulin resistance than ISR. In that context, Edholm et al documented a $34 \%$ decline in intrahepatic fat (9.3 to 6.1\%) after 2 weeks of starting a low-calorie diet in morbidly obese women awaiting bariatric surgery. ${ }^{17}$ Thus, the early improvement in ICR may add further support for the potentially important role of intrahepatic fat in mediating changes in ICR.

Rapid adaptation of ICR over ISR has also been hypothesized to preserve $\beta$-cell function in states of insulin resistance. ${ }^{18}$ Thus, in insulin-resistant states, the decrease in ICR may spare or minimize the increase in ISR necessary to maintain hyperinsulinemia. While appealing, this theory does not seem applicable to the observed changes during weight loss. If the priority is to minimize ISR, ISR would be expected to decline prior to the increase in ICR during weight loss, rather than the converse.

\section{CHANGING ICR DOES NOT AFFECT INSULIN SENSITIVITY}

In contrast to the above interventions, all of which increase ICR, salsalate treatment has been shown to decrease ICR. ${ }^{19}$ Salsalate is a non-steroidal anti-inflammatory drug shown to improve glucose tolerance in patients with type 2 diabetes. ${ }^{20}$ As an anti-inflammatory drug, salsalate has been hypothesized to reduce subacute inflammation associated with insulin resistance and lead to increase in insulin sensitivity. $^{21}$ We studied the mechanism of salsalate in insulinresistant individuals without diabetes who were randomized to $3.5 \mathrm{~g}$ of salsalate versus placebo in a $2: 1$ ratio. $^{19}$ After 4 weeks, salsalate treatment had no effect on insulin action or secretion, but was associated with a decrease in ICR, an increase in plasma insulin concentration, and a decrease in fasting glucose concentration in insulin-resistant individuals without diabetes. The exact mechanism by 
Table 1 Changes (\%) in plasma insulin, ISR and ICR during graded infusions of intravenous glucose

\begin{tabular}{llll}
\hline & $\begin{array}{l}\text { Insulin AUC } \\
\text { pmol/L×4 hours }\end{array}$ & $\begin{array}{l}\text { ISR AUC } \\
\text { pmol/min } \times 4 \text { hours }\end{array}$ & $\begin{array}{l}\text { ICR } \\
\left(\mathrm{L} / \mathrm{min} / \mathbf{m}^{2}\right)\end{array}$ \\
\hline Rosiglitazone $^{11}$ & $\downarrow 41 \%$ & $\downarrow 21 \%$ & $\uparrow 21 \%$ \\
Weight loss $^{14}$ & $\downarrow 31 \%$ & $\downarrow 6 \% \mathrm{NS}$ & $\uparrow 42 \%$ \\
Salsalate $^{18}$ & $\uparrow 29 \%$ & $\downarrow 6 \% \mathrm{NS}$ & $\downarrow 23 \%$ \\
\hline
\end{tabular}

All measurements were conducted following graded infusions of intravenous glucose for 4 hours. ICR was calculated by dividing ISR AUC over insulin AUC Arrows represent direction of change after intervention. All \% changes were significant $(p<0.05)$ unless noted as NS.

AUC, area under the curve; ICR, insulin clearance rate; ISR, insulin secretion rate; NS, not significant.

which salsalate decreased ICR is unknown, but it raises the possibility that drugs might be identified that would be helpful in treatment of type 2 diabetes by directly decreasing ICR. Lastly, results of this salsalate study solidify the directionality of association between insulin resistance and ICR. Insulin sensitivity affects ICR but ICR does not change insulin sensitivity.

\section{SUMMARY}

Table 1 summarises and compares the changes in plasma insulin concentration, ISR, and ICR in the three interventions discussed in this presentation. These data emphasize the fact that ICR is not fixed, and can change significantly in response to both lifestyle and pharmacological manipulation associated with decrease in plasma insulin concentration. In fact, in the interventions discussed, the change in ICR was more often statistically significant than the change in ISR.

Throughout this discussion of the modulatory role of ICR, the relationship among insulin resistance, compensatory hyperinsulinemia, and changes in ISR and ICR has received considerable attention. Our current view of these complex interactions is illustrated in figure 2. Weight gain will increase the prevalence of insulin resistance, leading to both an increase in ISR and decrease in ICR. Whether or not the decrease in ICR is secondary to an increase in hepatic fat requires further study. In any event, the changes in ISR and ICR are complementary in increasing plasma insulin concentration, thereby preventing loss of glucose



Figure 2 Modulators of ICR and plasma insulin concentration. Insulin resistance increases insulin secretion rate and decreases insulin clearance rate to raise plasma insulin concentration. Weight gain and treatment with salsalate decrease insulin clearance whereas weight loss and treatment with thiazolidinediones increase insulin clearance. Plus (+) sign denotes stimulatory effects and negative $(-)$ sign, inhibitory effects. homeostasis. In contrast, salsalate also decreases ICR, but in this instance there appears to be no effect on either insulin sensitivity or ISR. However, the decrease in ICR is associated with an increase in plasma insulin concentration. Finally, both weight loss and thiazolidinedione (eg, rosiglitazone) compounds are able to increase insulin sensitivity.

Future work will need to delineate the molecular pathways mediating the changes in insulin catabolism in insulinresistant states. Insulin uptake into cells is mostly mediated by the insulin receptor. ${ }^{7}$ Although there is some debate, ${ }^{22}$ insulin concentrations, especially at high levels and during prolonged increase, are believed to reduce the ICR. This inverse relationship between insulin concentration and clearance is in line with our results showing decrease in ICR in states of insulin resistance and hyperinsulinemia (figure 1). Although not completely understood, high insulin concentrations may decrease internalization ${ }^{23}$ and/or downregulate insulin receptors. ${ }^{7}$ After receptormediated internalization, insulin processing and degradation are predominately controlled by insulin-degrading enzymes (IDE). ${ }^{7}$ The regulation of these enzymes is also not well characterized. IDE polymorphisms may affect risk for type 2 diabetes, ${ }^{24}$ and IDE inhibitors are being explored as a treatment for type 2 diabetes. $^{25}$ Therefore, better understanding of IDE and regulators of ICR will enhance our understanding of glucose and insulin homeostasis, and affect future treatment of type 2 diabetes.

Competing interests None declared.

Ethics approval Stanford Institutional Review Board.

Provenance and peer review Commissioned; externally peer reviewed.

\section{REFERENCES}

1 Yeni-Komshian H, Carantoni M, Abbasi F, et al. Relationship between several surrogate estimates of insulin resistance and quantification of insulinmediated glucose disposal in 490 healthy nondiabetic volunteers. Diabetes Care 2000;23:171-5

2 Jones $C N$, Pei $D$, Staris $P$, et al. Alterations in the glucose-stimulated insulin secretory dose-response curve and in insulin clearance in nondiabetic insulin-resistant individuals. J Clin Endocrinol Metab 1997;82:1834-8.

3 Pei $D$, Jones $C N$, Bhargava $R$, et al. Evaluation of octreotide to assess insulin-mediated glucose disposal by the insulin suppression test. Diabetologia 1994;37:843-5.

4 Kim MK, Reaven GM, Chen YD, et al. Hyperinsulinemia in individuals with obesity: role of insulin clearance. Obesity (Silver Spring) 2015:23:2430-4.

5 Korenblat KM, Fabbrini E, Mohammed BS, et al. Liver, muscle, and adipose tissue insulin action is directly related to intrahepatic triglyceride content in obese subjects. Gastroenterology 2008;134:1369-75.

6 Goto T, Onuma T, Takebe $\mathrm{K}$, et al. The influence of fatty liver on insulin clearance and insulin resistance in non-diabetic Japanese subjects. Int J Obes Relat Metab Disord 1995;19:841-5.

7 Duckworth WC, Bennett RG, Hamel FG. Insulin degradation: progress and potential. Endocr Rev 1998; 19:608-24.

8 Kotronen A, Vehkavaara S, Seppälä-Lindroos A, et al. Effect of liver fat on insulin clearance. Am J Physiol Endocrinol Metab 2007;293:E1709-15.

9 Strang BD, Bertics SJ, Grummer RR, et al. Relationship of triglyceride accumulation to insulin clearance and hormonal responsiveness in bovine hepatocytes. J Dairy Sci 1998;81:740-7.

10 Shanik MH, Xu Y, Skrha J, et al. Insulin resistance and hyperinsulinemia: is hyperinsulinemia the cart or the horse? Diabetes Care 2008;31(Suppl 2): S262-8.

$11 \mathrm{Kim}$ SH, Abbasi F, Chu JW, et al. Rosiglitazone reduces glucose-stimulated insulin secretion rate and increases insulin clearance in nondiabetic, insulin-resistant individuals. Diabetes 2005:54:2447-52.

12 Tiikkainen $M$, Häkkinen AM, Korsheninnikova $E$, et al. Effects of rosiglitazone and metformin on liver fat content, hepatic insulin resistance, insulin clearance, and gene expression in adipose tissue in patients with type 2 diabetes. Diabetes 2004:53:2169-76. 
13 Jones $\mathrm{CN}$, Abbasi F, Carantoni $\mathrm{M}$, et al. Roles of insulin resistance and obesity in regulation of plasma insulin concentrations. Am J Physiol Endocrinol Metab 2000;278:E501-8.

14 McLaughlin T, Abbasi F, Lamendola C, et al. Differentiation between obesity and insulin resistance in the association with C-reactive protein. Circulation 2002;106:2908 -12.

15 Svendsen PF, Jensen FK, Holst JJ, et al. The effect of a very low calorie diet on insulin sensitivity, beta cell function, insulin clearance, incretin hormone secretion, androgen levels and body composition in obese young women. Scand I Clin Lab Invest 2012;72:410-19.

16 Erdmann J, Kallabis B, Oppel U, et al. Development of hyperinsulinemia and insulin resistance during the early stage of weight gain. Am J Physiol Endocrinol Metab 2008;294:E568-75.

17 Edholm D, Kullberg J, Karlsson FA, et al. Changes in liver volume and body composition during 4 weeks of low calorie diet before laparoscopic gastric bypass. Surg Obes Relat Dis 2015;11:602-6.

18 Kim SP, Ellmerer M, Kirkman EL, et al. Beta-cell "rest" accompanies reduced first-pass hepatic insulin extraction in the insulin-resistant, fat-fed canine model. Am J Physiol Endocrinol Metab 2007;292:E1581-9.
19 Kim S, Liu A, Ariel D, et al. Effect of salsalate on insulin action, secretion, and clearance in nondiabetic, insulin-resistant individuals: a randomized, placebo-controlled study. Diabetes Care 2014;37:1-2.

20 Goldfine $A B$, Fonseca $V$, Jablonski $K A$, et al. The effects of salsalate on glycemic control in patients with type 2 diabetes: a randomized trial. Ann Intern Med 2010;152:346-57.

21 Fleischman A, Shoelson SE, Bernier R, et al. Salsalate improves glycemia and inflammatory parameters in obese young adults. Diabetes Care 2008:31:289-94.

22 Castillo MJ, Scheen AJ, Letiexhe MR, et al. How to measure insulin clearance. Diabetes Metab Rev 1994;10:119-50.

23 Jochen A, Hays J, Lee M. Kinetics of insulin internalization and processing in adipocytes: effects of insulin concentration. J Cell Physiol 1989;141: 527-34.

24 Karamohamed S, Demissie S, Volcjak J, et al. Polymorphisms in the insulin-degrading enzyme gene are associated with type 2 diabetes in men from the NHLBI Framingham Heart Study. Diabetes 2003;52:1562-7.

25 Tang WJ. Targeting insulin-degrading enzyme to treat type 2 diabetes mellitus. Trends Endocrinol Metab 2016:27:24-34. 